\title{
Cyclooxygenase-selective inhibition of prostanoid formation: transducing biochemical selectivity into clinical read-outs
}

\author{
Carlo Patrono, ${ }^{1}$ Paola Patrignani, ${ }^{1}$ and Luis A. García Rodríguez ${ }^{2}$ \\ ${ }^{1}$ Department of Medicine and Aging, University of Chieti G. D’Annunzio School of Medicine, Chieti, Italy \\ ${ }^{2}$ Centro Español de Investigación Farmacoepidemiológica, Madrid, Spain
}

Address correspondence to: Carlo Patrono, Cattedra di Farmacologia, Dipartimento di Medicina e Scienze dell'Invecchiamento, Università degli Studi G. D’Annunzio, Via dei Vestini, 31, 66013 Chieti, Italy.

Phone:39-0871-3556777; Fax: 39-0871-3556718; E-mail: cpatrono@unich.it.

J. Clin. Invest. 108:7-13 (2001). DOI:10.1172/JCI200113418.

The two isoforms of cyclooxygenase (COX) catalyze the initial step in the formation of biologically important prostanoids, such as prostaglandin $(\mathrm{PG}) \mathrm{E}_{2}$, and thromboxane (TX) $\mathrm{A}_{2}$, in a variety of pathophysiologic processes. These include modulation of the inflammatory reaction, gastrointestinal (GI) cytoprotection and ulceration, angiogenesis and cancer, hemostasis and thrombosis, renal hemodynamics, and progression of kidney disease. Thus, it is not surprising that drugs inhibiting the activity of COX isozymes may have desirable as well as untoward effects on a variety of human diseases.

Low-dose aspirin provides a paradigm of COXisozyme-selective and cell-specific inhibition, by virtue of its short half-life and its ability to inactivate COX irreversibly (1). Other nonsteroidal anti-inflammatory drugs (NSAIDs) lack these unique pharmacokinetic and pharmacodynamic features and do not usually achieve the same degree of persistent platelet COX-1 inhibition as is obtained with low-dose aspirin. The coxib drugs, such as rofecoxib and celecoxib, were developed to spare COX-1 activity in GI mucosa and platelets, and this is achieved more or less effectively depending upon the biochemical selectivity of the individual agents (2). There is no evidence that coxibs inhibit COX-2 at sites different from those normally inhibited by nonaspirin NSAIDs, although the functional consequences of COX-2 inhibition may vary in the face of unopposed COX-1 activity at sites of cell-cell interactions. Here, we discuss the beneficial and harmful consequences of selective COX-1 versus COX- 2 inhibition and suggest a mechanistic interpretation of their major clinical read-outs. A leading theme is that the extent and persistence of platelet COX-1 inhibition represent a major determinant of the efficacy and safety of the currently available classes of COX inhibitors.

It should be emphasized that the relationships between inhibition of COX-isozyme activity, reduced prostanoid formation, and changes in prostanoiddependent cell function in vivo are not necessarily linear (3). When measuring clinically relevant outcomes of COX inhibition, one should consider the low incidence rate of these outcomes in the general popula-

tion (Table 1). Moreover, only a fraction of these events are likely to be COX-dependent. Thus, the signal-to-noise ratio of the measurements made in clinical studies is often less than ideal to allow unequivocal mechanistic interpretations.

Preventing arterial thrombosis by COX- 1 inhibition Human platelets provide a notable exception to the interesting paradigm of a COX-2-dependent amplification loop (discussed by Fitzpatrick and Soberman, this Perspective series, ref. 4) that propagates prostanoid release from its focal origin to surrounding cells. Platelets achieve the same goal through COX1-dependent generation of $\mathrm{TXA}_{2}$, which initiates an amplification loop that propagates the initial activation signal to adjacent platelets by inducing further platelet activation and $\mathrm{TXA}_{2}$ formation. The rate of prostanoid release is highly modifiable in this setting and is largely driven by enhanced substrate availability. Indeed, the maximal biosynthetic capacity of human platelets to produce $\mathrm{TXA}_{2}$ when challenged in vitro by thrombin exceeds the actual rate of $\mathrm{TXA}_{2}$ biosynthesis in vivo by several thousandfold (5). The impressive ability of the platelet to modify its production of a bioactive molecule may explain, at least in part, the unusual requirement for virtually complete suppression of platelet COX-1 activity if functional impairment is to translate into clinical benefit (3).

While disease-restricted expression of COX-2 during inflammation and preneoplastic growth may help to

\section{Table 1}

Incidence rates of major events possibly prevented or caused by COX inhibitors, as assessed in observational studies among non-users

Event

Heart failure

Myocardial infarction

Upper Gl bleeding/perforation

Colorectal cancer

Acute renal failure
Incidence rate per 1,000 patient-years

$1-4$

$0.6-1.7$

$0.4-0.7$

$0.02-0.08$ 
separate the participation of COX-1 in physiological processes and COX-2 in pathological processes (4), it is not yet clear how COX-1-dependent propagation of platelet activation can carry out its physiological role (primary hemostasis) without permitting the uncontrolled progression that would cause arterial thrombosis. The factors responsible for the localization and termination of the process or its unrestricted propagation most likely include the nature and duration of the pathophysiologic stimuli to platelet activation and the adequacy of the counterregulatory mechanisms evoked by platelet COX-1 activity. COX-2 induction in adjacent vascular endothelial cells may represent one such counterregulatory mechanism, one that may help amplify and prolong the antiplatelet signal initially evoked by COX-1-dependent $\mathrm{PGI}_{2}$ release at sites of platelet-vessel wall interaction.

A very large database of randomized clinical trials now offers the most compelling evidence that prevention of myocardial infarction and ischemic stroke by aspirin is largely due to permanent inactivation of platelet COX-1. These studies, which tested the efficacy and safety of the drug when given at daily doses ranging from as low as $30 \mathrm{mg}$ to as high as $1,500 \mathrm{mg}$ (6), have established two important facts. First, the antithrombotic effect of aspirin is saturable at doses in the range of $75-100 \mathrm{mg}$, as expected from ex vivo studies of the dose requirement for platelet COX-1 inactivation (1). Second, despite a half-life of approximately 20 minutes in the human circulation, the antithrombotic effect of aspirin is observed with dosing intervals of 24-48 hours, reflecting the permanent nature of platelet COX-1 inactivation and the duration of $\mathrm{TXA}_{2}$ suppression following oral dosing in humans (1). Other mechanisms of action that have been suggested to contribute to the antithrombotic effect of aspirin, such as an anti-inflammatory effect of the drug, are simply incompatible with these requirements.

Although the search for the lowest effective dose of aspirin for platelet inhibition was largely driven by the explicit concern of concomitant inhibition of vascular $\mathrm{PGI}_{2}$ production (7), it is still uncertain whether dosedependent suppression of the latter attenuates the antithrombotic effect of aspirin in clinical syndromes of vascular occlusion. The biochemical selectivity of low-dose aspirin arises from both pharmacokinetic determinants, such as the acetylation of platelet COX-1 that occurs in portal blood (prior to first-pass metabolism), and pharmacodynamic determinants, such as the limited sensitivity of endothelial COX-2 to the drug (6). Aspirin is an effective antithrombotic agent in a wide range of daily doses. However, both indirect comparisons of trials using different doses of aspirin in a variety of vascular disorders (8) and a recent randomized comparison of doses ranging from 81 to $1,300 \mathrm{mg}$ in patients undergoing carotid endarterectomy (9) suggest an inverse relationship between the aspirin daily dose and the relative risk reduction in vascular events. This effect is consistent with dose-dependent inhibition by aspirin of a mediator of thromboresistance.

Aspirin's unique features in inhibiting platelet COX-1 - its ability to inactivate the enzyme permanently through a short-lived active moiety (10) - are ideally suited to its role as an antiplatelet drug, because they severely limit the extent and duration of extraplatelet effects of the drug, including the inhibition of $\mathrm{PGI}_{2}$. Moreover, the cumulative nature of platelet COX-1 acetylation by repeated low doses of aspirin (11) explains the clinical efficacy of doses as low as $30-50 \mathrm{mg}$ daily, the predictable, high-grade inhibition of platelet $\mathrm{TXA}_{2}$ biosynthesis, and the persistence of the drug's effect. These features, in turn, may limit the consequences of less-than-ideal compliance in a real-world setting.

The "hit-and-run" mechanistic features of aspirin are shared by another successful antiplatelet drug, clopidogrel (6). This agent permanently inactivates the platelet ADP receptor P2Y12 through a short-lived active metabolite and is at least as effective as aspirin in preventing the vascular complications of atherothrombosis (6). In contrast, the incomplete and reversible inhibition of platelet COX-1 by nonaspirin NSAIDs or of GPIIb/IIIa by oral blockers of this platelet receptor is not associated with clinically detectable benefits, despite dose-dependent increase in bleeding complications (6). This apparent paradox may be reconciled by considering the different time requirements for highgrade blockade of these platelet proteins to prevent thrombosis in response to sudden fissuring of an atherosclerotic plaque vis-à-vis causing bleeding from a pre-existing GI lesion.

Permanent inactivation of platelet COX-1 by aspirin may lead to the prevention of thrombosis as well as to excess bleeding. At least two distinct COX-1-dependent mechanisms contribute to the increased risk of upper GI bleeding associated with aspirin exposure: inhibition of $\mathrm{TXA}_{2}$-mediated platelet function and impairment of $\mathrm{PGE}_{2}$-mediated cytoprotection in the GI mucosa (6). Whereas the former effect is dose-independent, at least for daily doses higher than $30 \mathrm{mg}$, the latter effect is clearly dose-dependent. Inhibition of platelet function is largely responsible for the twofold increase in the risk of upper GI bleeding associated with daily doses of aspirin in the range of $75-100 \mathrm{mg}$, inasmuch as a similar relative risk is associated with other antiplatelet agents that do not act on COX (12). Inhibition of COX-1-dependent cytoprotection amplifies bleeding risk by causing new mucosal lesions and is associated with a relative risk of 4-6 at the higher, analgesic or anti-inflammatory doses of aspirin. Assessing the net effect of aspirin requires an estimation of the absolute risk of the individual patient for thrombotic or hemorrhagic complications. In individ- 
uals at very low risk for vascular occlusion (i.e., $1 \%$ per year), a very small absolute benefit may be offset by exposure of very large numbers of healthy subjects to undue bleeding complications. As the risk of experiencing a major vascular event increases, so does the absolute benefit of antiplatelet prophylaxis with aspirin and, above a certain threshold, benefit clearly outweighs risk of bleeding (6).

\section{Preventing gastroduodenal ulcers and bleeding complications by COX-1 sparing}

The "COX-2 hypothesis" proposed that at comparable COX-2 inhibiting doses, highly selective COX-2 inhibitors would be as effective as traditional NSAIDs and cause less GI adverse effects putatively due to COX-1 inhibition. Testing this hypothesis required developments on two fronts. First, it was clearly necessary to develop adequate pharmacologic tools drugs that would spare COX-1 activity in clinically relevant targets (GI mucosa and platelets) at therapeutic plasma levels. Second, appropriate clinical end-points were needed that unequivocally reflect COX1-dependent GI toxicity. As seen in Table 2, several structurally different inhibitors are now available that afford a variable degree of biochemical selectivity for COX-2, as measured in vitro using human whole blood assays of COX-isozyme activity (13-16). Clinical trials designed to test the expected GI safety of these novel compounds have focused mainly on three classes of clinical end-points, none of which is ideally suited to the task. GI symptoms such as dyspepsia have a poor signal-to-noise ratio, and the COX-1 dependence of the signal is uncertain; these symptoms also fail to correlate with the presence of detectable GI lesions. Endoscopically detectable lesions, the second major class of end-point for these studies, have a favorable signal-to-noise ratio, and, while it has been established that they are largely COX-1-dependent, it remains uncertain whether they are reliable predictors of serious GI complications. Finally, some studies have attempted to measure these serious complications directly, tallying either perforations, ulcers, and bleeds (PUBs) or perforations, obstructions, and bleeds (POBs). These measures have an uncertain signal-to-noise ratio, because of lack of adequately sized placebo-controlled studies. Moreover, the hemorrhagic nature of the most prevalent component of these combined end-points makes it likely that they reflect primarily GI complications related to inhibition of platelet COX-1. Because of the low event rate, very large sample sizes or prolonged drug exposure are needed in order to detect differences between drugs (2).

Celecoxib and rofecoxib represent a new World Health Organization class of NSAIDs that were developed to test the COX-2 hypothesis. McAdam et al. demonstrated dose-dependent inhibition of platelet COX-1 after single doses of celecoxib (up to $800 \mathrm{mg}$ ), with large interindividual variability in pharmacodynamic response ranging between $10 \%$ and $80 \%$ inhibition at the highest plasma levels (17). Two endoscopy trials demonstrated a significantly lower incidence of endoscopic ulcers in patients treated with celecoxib than in those receiving NSAIDs (diclofenac or naproxen). A pooled analysis of 14 randomized trials in approximately 11,000 patients suggested a $0.2 \%$ yearly rate of ulcer complications (POBs) in patients receiving various doses of celecoxib, as compared with $1.7 \%$ in patients treated with NSAIDs. The design of the GI outcome study, CLASS, was such that it could reliably detect a $75 \%$ reduction in relative risk for upper GI ulcer complications. However, because of the relatively short duration of the study and higher-than-expected event rate observed in the celecoxib group, the rates of ulcer complications associated with celecoxib $(0.76 \%)$ and with NSAIDs $(1.45 \%)$ were statistically indistinguishable in this study (18).

In contrast, two independent studies of the related drug rofecoxib in healthy subjects and in patients with rheumatoid arthritis (RA) showed no evidence of any detectable change in platelet COX-1 activity after a single dose of up to $1,000 \mathrm{mg}$, or following repeated dosing with $50 \mathrm{mg}$ daily, respectively. Endoscopy trials demonstrated a significantly lower incidence of endoscopic ulcers in patients treated with rofecoxib than in those receiving the NSAID ibuprofen. A combined analysis of eight randomized studies in approximately 5,000 subjects with osteoarthritis suggested a 50\% reduction in the incidence of clinically important upper GI events in those receiving rofecoxib rather than NSAIDs. The GI

\section{Table 2}

Biochemical selectivity of currently available COX-2 inhibitors, as measured in vitro with human whole blood assays of COX-isozyme activity

$\begin{array}{cc}\text { Inhibitor } & \text { COX-1/COX- } \\ & \mathrm{IC}_{50} \text { ratio } \\ \text { Ibuprofen } & 0.5 \\ \text { Naproxen } & 0.7 \\ \text { 6-MNA } & 1.5 \\ \text { Acetaminophen } & 1.6 \\ \text { Indomethacin } & 1.9 \\ \text { Meloxicam } & 18 \\ \text { Nimesulide } & 19 \\ \text { Diclofenac } & 29 \\ \text { Celecoxib } & 30 \\ \text { Rofecoxib } & 267\end{array}$

$I C_{50}$ values for the inhibition of platelet COX-1 and monocyte COX- 2 were obtained as described $(13,29)$. A substantially similar rank order of selectivity has been reported by other investigators using the same methodology (14-16). 6-MNA, 6-methoxy-2 naphthyl-acetic acid 


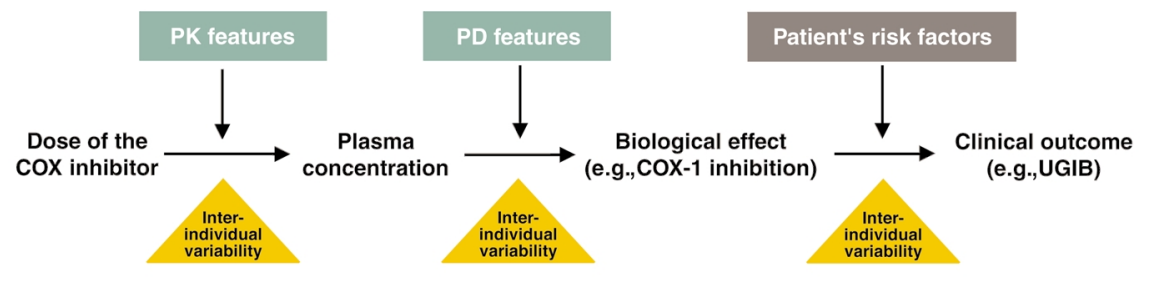

b

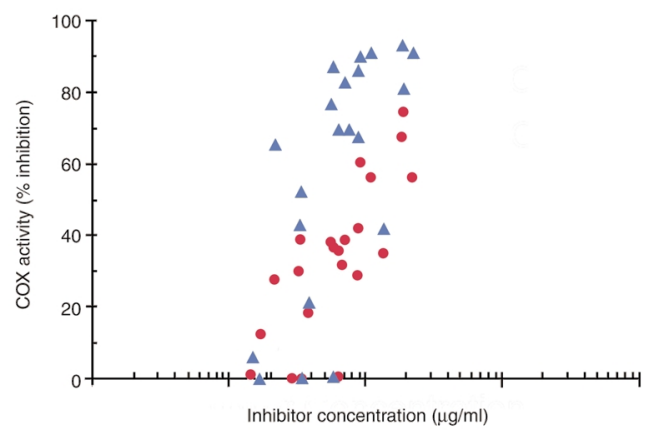

C

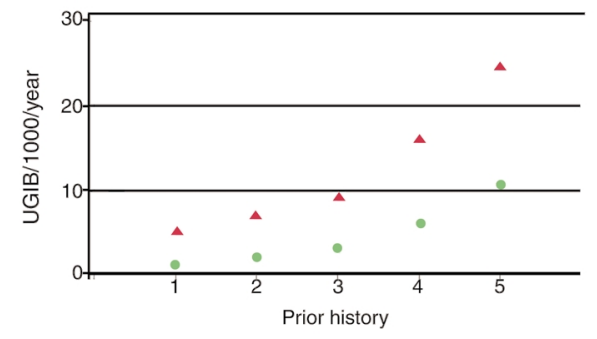

\section{Figure 1}

Determinants and sources of variability in GI complications of COX-inhibitory drugs. (a) Major determinants of the likelihood of an upper GI bleeding (UGIB) complication resulting from administration of a COX inhibitor include pharmacokinetic (PK) and pharmacodynamic (PD) variables, as well as the interaction of the drug with pre-existing risk factors for UGIB. PK features, such as half-life of the drug, and PD features, such as its selectivity for the COX-2 isoform, are intrinsic to the COX inhibitor. The presence or absence of risk factors for UGIB will clearly vary between patients. Significant interindividual variability arises from several sources, as shown in $\mathbf{b}$ and $\mathbf{c}$, and is superimposed on each of these effects. Modified from ref. 2. (b) In addition to the variable plasma levels achieved after oral dosing of a COX inhibitor, the inhibition of the platelet COX-1 isozyme (circles) and the monocyte COX-2 isozyme (triangles) in response to any given plasma level of the inhibitor is highly variable between subjects. COXisozyme inhibition, measured ex vivo, is plotted as a function of plasma meloxicam levels in individual subjects. Modified from ref. 31. (c) The variable excess of UGIB induced by NSAIDs as a function of the increasing severity of prior history: 1, none; 2, dyspepsia; 3, gastritis; 4, uncomplicated ulcer; 5, complicated ulcer. Within each cohort of subjects, UGIB rates for individuals currently using NSAIDs (triangles) exceed those for non-users (circles), but the effect of prior history greatly amplifies that of NSAID use (data from ref. 20).

outcome study, VIGOR, showed that the rate of confirmed clinical GI events was halved by rofecoxib as compared with naproxen in RA patients (19). The results of VIGOR are consistent with the earlier combined analysis of smaller trials and suggest a substantial risk reduction for PUBs. Together, these studies provide the strongest evidence to date for the clinical relevance of the COX-2 hypothesis in terms of improved GI safety. Given that the vast majority of ulcer complications are hemorrhagic in nature, it is likely that the statistically significant $60 \%$ relative risk reduction in POBs associated with rofecoxib as compared with naproxen was mainly related to sparing of platelet COX-1 even at very high plasma levels of rofecoxib. The failure of CLASS to demonstrate a statistically significant difference between celecoxib and comparator NSAIDs with respect to the rates of complicated GI events might reflect inadequate biochemical selectivity of celecoxib (as reflected by highly variable dose-dependent inhibition of platelet COX-1 ex vivo), as well as the smaller number of GI complications seen in the CLASS trial than in the
VIGOR trial $(18,19)$. The similar biochemical selectivity of celecoxib and one of the two NSAID comparators, diclofenac (Table 2), as well as the concomitant use of low-dose aspirin in about one-fifth of the patients, represents additional confounding factors for the CLASS trial.

In summary, COX-1/COX-2 selectivity does indeed translate to improved GI safety, at least when the drug employed inhibits COX-2 but not COX-1 at therapeutic plasma levels, and when the study enjoys adequate statistical power to detect biologically plausible differences in the occurrence of relatively rare events. However, as illustrated in Figure 1, biochemical selectivity is but one of several important determinants of the risk of experiencing a serious GI complication during long-term NSAID therapy (2). Interindividual variability in drug plasma levels as well as in the corresponding pharmacodynamic response, in addition to known risk factors for these GI complications (20), should all be considered in this context. The apparent dissociation between phase III endoscopic findings and phase IV outcome trials may be reconciled by postulating that gastroduodenal lesions 
develop as a consequence of persistent, moderate inhibition of mucosal COX-1 activity in a large proportion of exposed patients, while bleeding complications occur as a result of transient, high-grade inhibition of platelet COX-1 in a very small percentage of exposed patients. In terms of probability, the wider the separation between COX-2 and COX-1 dose-response curves of the inhibitor (an index of biochemical selectivity), the lower the proportion of exposed patients who may eventually experience a clinically meaningful inhibition of platelet COX1 , because of an unusually high drug level or an unusually intense pharmacodynamic response to a usual drug level. The failure - so far - of other NSAIDs to confirm the COX-2 hypothesis is likely to reflect a combination of limited biochemical selectivity and inadequate statistical power of the trials to detect a moderate effect on the most serious outcomes. While the results of large-scale clinical studies performed with meloxicam and celecoxib are promising and suggest a trend toward improved GI safety associated with less-than-ideal biochemical selectivity, they fail to make the point in statistically convincing terms (2).

\section{Preventing colorectal cancer by COX-1 or COX-2 inhibition?}

There is a large body of epidemiologic evidence suggesting that regular users of aspirin and nonaspirin NSAIDs have a reduced risk of colorectal cancer (21). This is complemented by murine studies of chemically induced intestinal carcinogenesis and genetically engineered models of intestinal polyposis, both of which are sensitive to changes in COX-isozyme expression or activity (ref. 22; see also Fitzpatrick and Soberman, this Perspective series, ref. 4). One proposed mechanism for this type of chemoprevention is the inhibition of COX-2 in intestinal epithelial cells, stromal cells, or endothelial cells of newly formed blood vessels (22). COX-2 is overexpressed in most colonic cancers and in polyps of patients with familial adenomatous polyposis (FAP). Subsequently, COX-2 has been found to be expressed in a wide variety of epithelial tumors. It is unclear how suppression of COX-2 expression or activity might restore antitumor reactivity, but the ability of prostanoids to influence cellular proliferation and apoptosis and modulate cytokine synthesis suggests some testable hypotheses. Prostanoids may also modulate the nuclear translocation and function of tumor suppressor gene products. In the early stages of intestinal carcinogenesis, COX-2 appears to be expressed predominantly in stromal cells, but it is later detectable in surrounding inflammatory cells, in the carcinomatous epithelial cells, and in the endothelial cells of new blood vessels, where it may play an additional role in angiogenesis (23).

The only evidence from randomized clinical trials that supports the COX-2 dependence of the early stages of intestinal carcinogenesis is related to FAP patients, in whom both a conventional NSAID, sulindac, and celecoxib reversibly suppress intestinal polyps (21). This type of evidence is complemented by eight case-control and cohort studies suggesting a $30-50 \%$ decrease in the risk of colorectal adenoma among long-term users of aspirin or nonaspirin NSAIDs (24). Although no single randomized study has yet tested the COX-2 hypothesis with colorectal cancer as the primary end-point, 17 out of 18 epidemiologic studies that specifically examined the matter found that regular use of aspirin and/or nonaspirin NSAIDs reduced the risk of colorectal cancer by close to $50 \%(25)$.

It is interesting to note that the benefit of aspirin is apparent at dosing intervals of $\geq 24$ hours as well as at relatively low daily doses that are unlikely to produce substantial inhibition of COX-2. These considerations also greatly diminish the likelihood of COX-independent effects of aspirin contributing to the apparent protection against colorectal cancer, because of the markedly different duration and concentration dependence of these effects (e.g., inhibition of IKB kinase) vis-à-vis COX inhibition. Perhaps more important than the dose is the dosing interval of aspirin intake, given the 15- to 20minute half-life of the drug in the human circulation. The apparent protection against both colorectal adenoma and carcinoma, recently described $(24,25)$ in association with once-a-day aspirin regimens, raises the intriguing possibility that permanent inactivation of platelet COX-1 restores antitumor reactivity. This working hypothesis is apparently at odds with the proposed COX-2 dependence of early intestinal carcinogenesis, but the models could be reconciled if it could be shown that activated platelets signal COX-2 upregulation in one or more cell types involved in tumor induction and/or angiogenesis. Such a platelet-dependent effect might work through paracrine mediators, either lipids or proteins. The finding that deletion of either the COX1 or the COX2 gene reduces both polyp formation in the Min mouse and chemically induced skin carcinogenesis (ref. 26; see also Fitzpatrick and Soberman, this Perspective series, ref. 4) is compatible with such a sequential paradigm. A head-to-head comparison of low-dose aspirin and a highly selective coxib would be required to test this hypothesis in humans. It is likely that, in addition to any chemopreventive effect, the differential impact of these pharmacologic probes on the cardiovascular and bleeding risks of the exposed patients will substantially shape the benefit/risk profiles of these different preventive strategies.

Does COX-1/COX-2 selectivity affect the consequences of COX-2 inhibition?

There is no compelling evidence that the available coxibs inhibit COX-2 more effectively than do conven- 
tional NSAIDs or that they act on a different pool of COX-2. However, it is unclear whether the efficacy or safety of coxibs may be affected by unopposed COX-1 activity. This may have clinical implications in at least three areas: pain relief, renal function, and thromboresistance of the vessel wall.

The difficulty in assessing the contribution of unopposed COX-1-dependent prostanoid formation, when comparing a highly selective coxib with a nonselective NSAID, relates to the poor signal-to-noise ratio or the low incidence of the clinical read-outs of COX-2 inhibition. Thus, although the analgesic efficacy of celecoxib and rofecoxib appears indistinguishable from that of several NSAID comparators, both in acute-pain models and in long-term studies of patients with osteoarthritis (2), the specificity and sensitivity of the measurable clinical end-points is largely inadequate to detect small differences in efficacy that might reflect the participation of COX-1-derived prostanoids in hyperalgesia (27). Similarly, the relatively low incidence of renal effects related to COX-2 inhibition, such as peripheral edema and worsening of pre-existing hypertension (28), would require both very large sample sizes and accurate recording in order to reliably detect differences (between coxibs and NSAIDs, for example, or between celecoxib and rofecoxib) that might reflect differential inhibition of COX-1 activity within the kidney. Moreover, any meaningful comparison to this effect would require testing different drugs at the same level of COX-2 inhibition, as measurable ex vivo in circulating monocytes (29). A different pattern of renal effects might carry substantial clinical relevance as a potential determinant of the relative risk of developing heart failure. It should be noted that the excess burden of heart failure due to NSAIDs is at least as great as, and possibly greater than, that due to GI bleeding complications (30).

Finally, whether the vascular consequences of endothelial COX-2 inhibition are modified by unopposed platelet $\mathrm{TXA}_{2}$ production is currently being debated on the basis of the controversial cardiovascular findings of the VIGOR trial (19). In this study, the rate of myocardial infarction was significantly different between patients treated with rofecoxib $(0.4 \%)$ and those treated with naproxen $(0.1 \%)$. In contrast, the same rate $(0.3 \%)$ was found in both celecoxib- and NSAID-treated patients in the CLASS trial (18). The low cardiovascular risk $(<1 \%$ per year) and short follow-up of patients recruited in both the VIGOR and CLASS trials ( 9 and 6 months, respectively) make detection of a moderate difference (e.g., $25 \%$ ) in major vascular events between coxibs and conventional NSAIDs a rather questionable exercise if based on individual trial data. An independent overview of all coxib trials by a trialists' collaborative group appears to offer a feasible strategy to answer this question, one that would not require a very large head-to-head randomized trial with vascular endpoints or additional epidemiologic studies.

As a second wave of highly selective COX-2 inhibitors, such as valdecoxib, etoricoxib, and COX-189, moves towards approval, it seems appropriate that the medical-scientific community contributes to redefining a research agenda that incorporates the above considerations and concerns.

\section{Acknowledgments}

This work was supported by a grant from the Italian Ministry of University and Research (MURST) to the Center of Excellence on Aging. The expert editorial assistance of Daniela Basilico is gratefully acknowledged.

1. Patrono, C. 1994. Aspirin as an antiplatelet drug. N. Engl. J. Med. 330: 1287-1294.

2. FitzGerald, G.A., and Patrono, C. 2001. The anti-inflammatory properties and safety of coxibs, selective inhibitors of cyclooxygenase-2. $N$. Engl. J. Med. In press.

3. Reilly, I.A.G., and FitzGerald, G.A. 1987. Inhibition of thromboxane formation in vivo and ex vivo: implications for therapy with platelet inhibitory drugs. Blood. 69:180-186.

4. Fitzpatrick, F.A., and Soberman, R. 2001. Regulated formation of eicosanoids. J. Clin. Invest. 107:1347-1351.

5. Patrono, C., et al. 1986. Estimated rate of thromboxane secretion into the circulation of normal man. J. Clin. Invest. 77:590-594.

6. Patrono, C., et al. 2001. Platelet-active drugs: the relationships among dose, effectiveness, and side effects. Chest. 119:39S-63S.

7. FitzGerald, G.A., et al. 1983. Endogenous biosynthesis of prostacyclin and thromboxane and platelet function during chronic administration of aspirin in man. J. Clin. Invest. 71:678-688.

8. Antithrombotic Trialists' Collaboration. 2001. Prevention of death, myocardial infarction and stroke by antiplatelet therapy: collaborative meta-analysis of 266 trials involving 200,000 patients at high risk of occlusive vascular events. BMJ. In press.

9. Taylor, D.W., et al. 1999. Low-dose and high-dose acetylsalicylic acid for patients undergoing carotid endarterectomy: a randomised controlled trial. Lancet. 355:1295-1305.

10. Roth, G.J., Stanford, N., and Majerus, P.W. 1975. Acetylation of prostaglandin synthase by aspirin. Proc. Natl. Acad. Sci. USA. 72:3073-3077.

11. Patrignani, P., Filabozzi, P., and Patrono, C. 1982. Selective cumulative inhibition of platelet thromboxane production by low-dose aspirin in healthy subjects. J. Clin. Invest. 69:1366-1372.

12. García Rodríguez, L.A., et al. 1998. Risk of hospitalization for upper gastrointestinal tract bleeding associated with ketorolac, other nonsteroidal anti-inflammatory drugs, calcium antagonists, and other antihypertensive drugs. Arch. Intern. Med. 158:33-39.

13. Patrignani, P., et al. 1997. Differential inhibition of human prostaglandin endoperoxide synthase- 1 and -2 by nonsteroidal antiinflammatory drugs. J. Physiol. Pharmacol. 48:623-631.

14. Chan, C.-C., et al. 1999. Rofecoxib [Vioxx, MK-0966; 4-(4'-Methylsulfonylphenyl)-3-phenyl-2-(5H)-furanone]: a potent and orally active cyclooxygenase-2 inhibitor. Pharmacological and biochemical profiles. J. Pharmacol. Exp. Ther. 290:551-560.

15. Warner, T.D., et al. 1999. Nonsteroid drug selectivities for cyclo-oxygenase- 1 rather than cyclo-oxygenase- 2 are associated with human gastrointestinal toxicity: a full in vitro analysis. Proc. Natl. Acad. Sci. USA. 96:7563-7568.

16. Cryer, B., and Feldman, M. 1998. Cyclooxygenase-1 and cyclooxygenase2 selectivity of widely used nonsteroidal anti-inflammatory drugs. Am. J. Med. 104:413-421.

17. McAdam, B.F., et al. 1999. Systemic biosynthesis of prostacyclin by cyclooxygenase (COX)-2: the human pharmacology of a selective inhibitor of COX-2. Proc. Natl. Acad. Sci. USA. 96:272-277.

18. Silverstein, F.E., et al. 2000. Gastrointestinal toxicity with celecoxib vs nonsteroidal anti-inflammatory drugs for osteoarthritis and rheumatoid arthritis. The CLASS study: a randomized controlled trial. JAMA. 284:1247-1254. 
19. Bombardier, C., et al. 2000. Comparison of upper gastrointestinal toxicity of rofecoxib and naproxen in patients with rheumatoid arthritis. VIGOR study group. N. Engl. J. Med. 343:1520-1528.

20. García Rodríguez, L.A., and Hernández-Díaz, S. 2001. The relative risk of upper gastrointestinal complications among users of acetaminophen and non-steroidal anti-inflammatory drugs. Epidemiology. In press.

21. Janne, P.A., and Mayer, R.J. 2000. Chemoprevention of colorectal cancer. N. Engl. J. Med. 342:1960-1968.

22. Prescott, S.M., and Fitzpatrick, F.A. 2000. Cyclooxygenase-2 and carcinogenesis. Biochim. Biophys. Acta. 1470:M69-M78.

23. Prescott, S.M. 2000. Is cyclooxygenase- 2 the alpha and the omega in cancer? J. Clin. Invest. 105:1511-1513.

24. García Rodríguez, L.A., and Huerta-Alvarez, C. 2000. Reduced incidence of colorectal adenoma among long-term users of nonsteroidal antiinflammatory drugs: a pooled analysis of published studies and a new population-based study. Epidemiology. 11:376-381.

25. García Rodríguez, L.A., and Huerta-Alvarez, C. 2001. Reduced risk of colorectal cancer among long-term users of aspirin and non-aspirin nonsteroidal anti-inflammatory drugs. Epidemiology. 12:88-93.

26. Chulada, P.C., et al. 2000. Genetic disruption of Ptgs-1, as well as Ptgs2 , reduces intestinal tumorigenesis in Min mice. Cancer Res. 60:4705-4708.

27. McAdam, B.F., et al. 2000. Regulated expression of the two cyclooxygenase isoforms in a human syndrome of inflammation. J. Clin. Invest. 105:1473-1482.

28. Brater, D.C., Harris, C., Redfern, J.S., and Gertz, B.J. 2001. Renal effects of COX-2 selective inhibitors. Am. J. Nephrol. 21:1-15.

29. Patrignani, P., et al. 1994. Biochemical and pharmacological characterization of the cyclooxygenase activity of human blood prostaglandin endoperoxide synthases. J. Pharmacol. Exp. Ther. 271:1705-1712.

30. Heerdink, E.R., et al. 1998. NSAIDs associated with increased risk of congestive heart failure in elderly patients taking diuretics. Arch. Intern. Med. 158:1108-1112.

31. Panara, M.R., et al. 1999. Dose-dependent inhibition of platelet cyclooxygenase- 1 and monocyte cyclooxygenase- 2 by meloxicam in healthy subjects. J. Pharmacol. Exp. Ther. 290:276-280. 